\title{
Joint observations of a traveling ionospheric disturbance with the Paratunka OMTI camera and the Hokkaido HF radar
}

\author{
A. Koustov ${ }^{1,2}$, N. Nishitani ${ }^{2}$, P. V. Ponomarenko ${ }^{1}$, K. Shiokawa ${ }^{2}$, S. Suzuki ${ }^{3}$, B. M. Shevtsov ${ }^{4}$, and J. W. MacDougall ${ }^{5}$ \\ ${ }^{1}$ Institute of Space and Atmospheric Studies, University of Saskatchewan, 116 Science Place, Saskatoon, S7N 5E2 Canada \\ ${ }^{2}$ Solar-Terrestrial Environment Laboratory, Nagoya University, Nagoya, Japan \\ ${ }^{3}$ Department of Information and Communication Engineering, University of Electro-Communications, Tokyo, Japan \\ ${ }^{4}$ Institute of Cosmophysical Research and Radio Wave Propagation (IKIR) of FEB Russian Academy of Sciences, \\ Paratunka, Russia \\ ${ }^{5}$ Department of Electrical Engineering, University of Western Ontario, London, Ontario, Canada
}

Received: 7 October 2008 - Revised: 28 April 2009 - Accepted: 27 May 2009 - Published: 9 June 2009

\begin{abstract}
On 10 September 2007 between 10:00 and 14:00 UT, the OMTI all-sky imager at Paratunka (Kamchatka, Russia, GLAT $\sim 52^{\circ}$ ) observed the onset and southwestern progression of a localized depletion region in the airglow intensity. The perturbation, while being stretched in the NW-SE direction, crossed the entire field of view of the camera. During the event, the Hokkaido SuperDARN HF radar was monitoring echoes in the Paratunka longitudinal sector. It was detecting a localized band of ground scatter echoes progressing equatorward synchronously with the motion of the optical perturbation. It is suggested that both features resulted from the onset and south-western progression of a localized region with enhanced electric field that influenced the distribution of the plasma density in the ionosphere. Modeling of the HF ground scatter dynamics based on numerical ray tracing demonstrated qualitative consistency with the observations.
\end{abstract}

Keywords. Ionosphere (Ionospheric disturbances; Midlatitude ionosphere; Wave propagation)

\section{Introduction}

Over the last decade, Super Dual Auroral Radar Network (SuperDARN) HF radars have been successfully used for monitoring plasma convection at high latitudes by measuring Doppler shift of radio waves back-scattered by small-scale ionospheric irregularities (Greenwald et al., 1995; Chisham

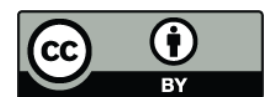

Correspondence to: A. Koustov (sasha.koustov@usask.ca) et al., 2007). From the beginning of the project, however, it has been anticipated that other types of echoes would also be useful for ionospheric studies (Greenwald et al., 1995), including the so-called ground scatter (GS) echoes. These echoes occur when radio waves are refracted or reflected by the ionosphere toward the Earth's surface, which then scatters part of their energy back to the receiver along the same path.

The GS echoes in SuperDARN records are identified as low velocity $(<20 \mathrm{~m} / \mathrm{s})$ and narrow spectral width $(<50 \mathrm{~m} / \mathrm{s})$ returns, although over the years the initial simple criteria have been modified to handle a mixture of ground and ionospheric scatter (e.g., Ponomarenko et al., 2008). GS echoes are usually strong and persist for hours, especially during the daytime when the ionosphere is dense and strong refraction/reflection of HF radio waves can be easily achieved. GS returns are often quasi-periodic and progressing in range; these are traditionally associated with gravity wave propagation through the ionosphere (e.g., Samson et al., 1989; Bristow et al., 1994; MacDougal et al., 2001). SuperDARN results in this area were recently summarized by Chisham et al. (2007).

Quite often, however, a solitary enhancement in the GS power is observed; it can remain at the same location for a long time or slowly move, typically in the equatorward direction. One possible interpretation of this phenomenon involves an onset of a localized ionospheric patch with modulated electron density, which can alter spatial structure of GS echoes through focusing/defocusing effects. As the patch progresses through the field of view of a radar, the resulting GS feature would be detected as shifting in range. In a case of the propagating ionospheric patch, one can say that

Published by Copernicus Publications on behalf of the European Geosciences Union. 


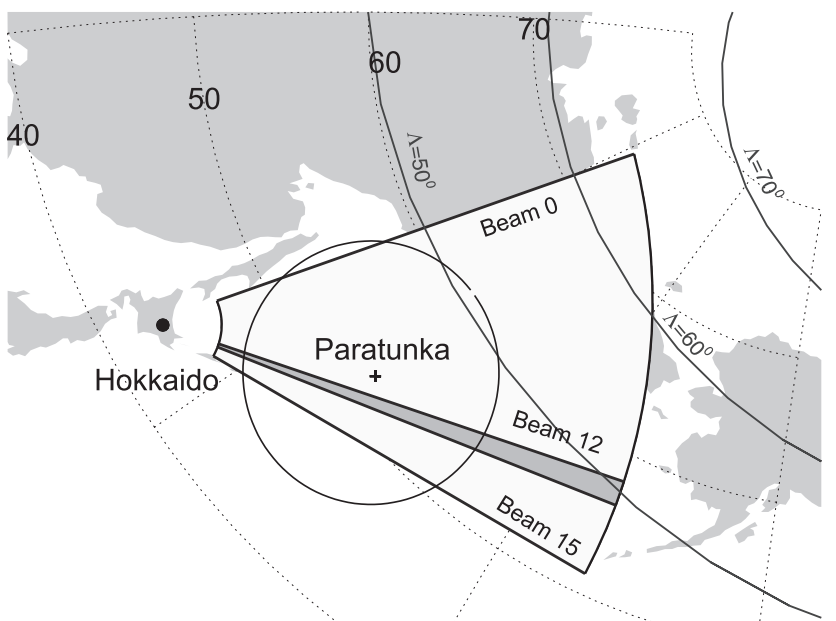

180

210

Fig. 1. Fields of view of the Hokkaido SuperDARN radar (between 400 and $3550 \mathrm{~km}$ ) and the OMTI all-sky camera at Paratunka (for the off-zenith angle of $75^{\circ}$ ). The dark shaded sector within the Hokkaido field of view indicates the orientation of beam 12; data for this beam are discussed in this study.

the HF radar detects a medium scale traveling ionospheric disturbance (MSTID). As a MSTID propagates through the ionosphere, localized vertical movements of the ionospheric plasma are also expected to occur. These movements would represent $\boldsymbol{E} \times \boldsymbol{B}$ plasma drifts caused by the polarization electric field associated with the patch (e.g., Perkins, 1973). Studies of HF radar signatures related to the localized propagating ionospheric perturbations using SuperDARN are currently limited to a recent paper by Nishitani et al. (2007).

MSTIDs can also be studied through optical measurements of airglow (e.g., Garcia et al., 2000; Shiokawa et al., 2003a). It is believed that in a localized area of the ionosphere with an enhanced electric field (of proper direction), the plasma is forced to move up (or down) so that the rates of chemical reactions change resulting in modulation of airglow intensity (e.g., Kelley and Makela, 2001; Shiokawa et al., 2003b). Since vertical motions of the ionospheric plasma can be studied by measuring the Doppler velocity of GS echoes, joint observations of GS echoes and airglow perturbations could be useful to get insights into mechanisms of MSTID formation.

The goal of this study is to analyze in detail simultaneous HF radar and airglow observations of a MSTID moving through the common field of view and to establish/clarify radar and optical signatures of a propagating localized structure with enhanced electric field.

\section{Experimental configuration}

Over the last decade, several airglow cameras, Optical Mesosphere Thermosphere Imagers (OMTIs), were installed by the Nagoya University at various locations (Shiokawa et al., 2009) as a part of a general effort to study global scale phenomena such as gravity wave generation, their propagation and effects in various atmospheric and ionospheric regions. In this study we consider data from an OMTI camera installed on the Russian territory at Paratunka, Kamchatka, (52.97 N, 158.25 E, dipole magnetic latitude: 45.8 N), Fig. 1. In addition, the camera is meant to enhance the scientific output from the Hokkaido SuperDARN HF radar. The radar is located near Rikubetsu, Northern Japan (Nishitani et al., 2005, 2007), Fig. 1. It is in operation since November 2006. Figure 1 shows that the Paratunka OMTI camera covers significant portion of the Hokkaido radar field of view (FoV) at short ranges.

\section{Observations}

Over the first half a year of joint observations, several interesting events have been found. Here we focus on an event of 10 September 2007, between 10:00 and 14:00 UT.

\subsection{Keogram of airglow perturbations along Hokkaido beam 12}

An OMTI all-sky camera records airglow images every 2 min. Optical signatures of MSTID can be identified from 2-D images, once the background luminosity level is removed from every frame. Since the goal of this study is to compare optical and radar data, it is more convenient to introduce the event by considering optical data along a specific radar beam, i.e. by a keogram. Figure 2 shows a Paratunka keogram for 630-nm airglow along the Hokkaido radar beam \#12. Here the top panel (a) shows the raw data in Rayleighs and the bottom panel (b) presents the percent deviation of the airglow intensity from 1-h running averages. The intensity deviation $\Delta I(t)$ is defined as $\Delta I(t)=\left(I(t)-I_{a}(t)\right) / I_{a}(t)$, where $I(t)$ and $I_{a}(t)$ are the raw airglow intensity at time $t$ and running average over $t= \pm 30 \mathrm{~min}$, respectively, for each pixel of the image. While optical features were observed along several radar beams, beam \#12 was deliberately chosen because the most prominent optical effect was observed along it. The missing data at the bottom of Fig. 2 correspond to the close-range cutoff of the camera field of view for the luminosity height of $\sim 250 \mathrm{~km}$. Paratunka's location at $\sim 1500 \mathrm{~km}$ from the radar is marked in Fig. 2 by a horizontal tick. The low airglow intensity, clearly seen between 1200 and $1800 \mathrm{~km}$ at all UT times, is caused by the Van Rhijn effect, i.e. the decrease of the light intensity at smaller zenith angles due to smaller light collecting volume. Finally, the vertical lines in Fig. $2 b$ mark the study interval analyzed in this work. 


\section{Paratunka OMTI: 10 Sept 2007 (along Hokkaido Beam 12)}

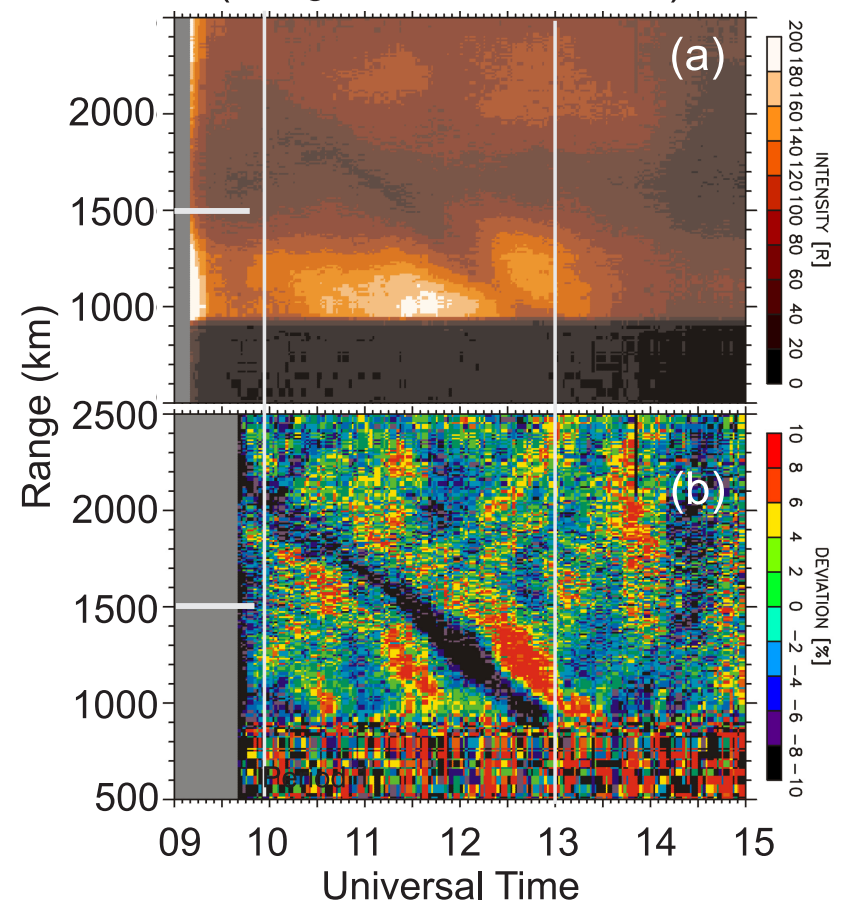

Fig. 2. (a) Keogram of the 630-nm airglow intensity as recorded by the Paratunka OMTI camera along the Hokkaido radar beam 12 . The luminosity height was assumed to be $250 \mathrm{~km}$. (b) The same data but reprocessed into the deviation of the airglow intensity from the 1-h running average background level (along the same beam 12).

A prominent feature of the data presented in Fig. 2a is a stripe of the reduced airglow intensity stretching diagonally across the entire keogram, from $\sim 2200 \mathrm{~km}$ at 10:00 UT to $\sim 1000 \mathrm{~km}$ at 13:00 UT. The area of reduced luminosity is clearer after the background is removed (Fig. 2b). The slope of the stripe is nearly constant during the study interval. The intensity depletion was strongest during 11:3013:00 UT, and the depletion moved away (equatorward) from the camera's FoV after $\sim 13: 30$ UT. Enhancement of airglow intensity equatorward and poleward of the stripe present (to significant extent) an artifact of the background removal procedure.

\subsection{Hokkaido radar data in beam 12}

Figure 3 summarizes the radar data for beam 12 during the study interval. Figure $3 \mathrm{a}$ shows the range-time plot for the echo intensity while Fig. $3 \mathrm{~b}$ and c shows the range-time plots for the Doppler velocity with two different velocity scales. In Fig. 3b, the Doppler velocity is indicated for all echoes and the scale is set to more easily identify the GS echoes using a grey color. In Fig. 3c, only ground scatter echoes are considered. The velocity scale in Fig. $3 \mathrm{c}$ is set to show the

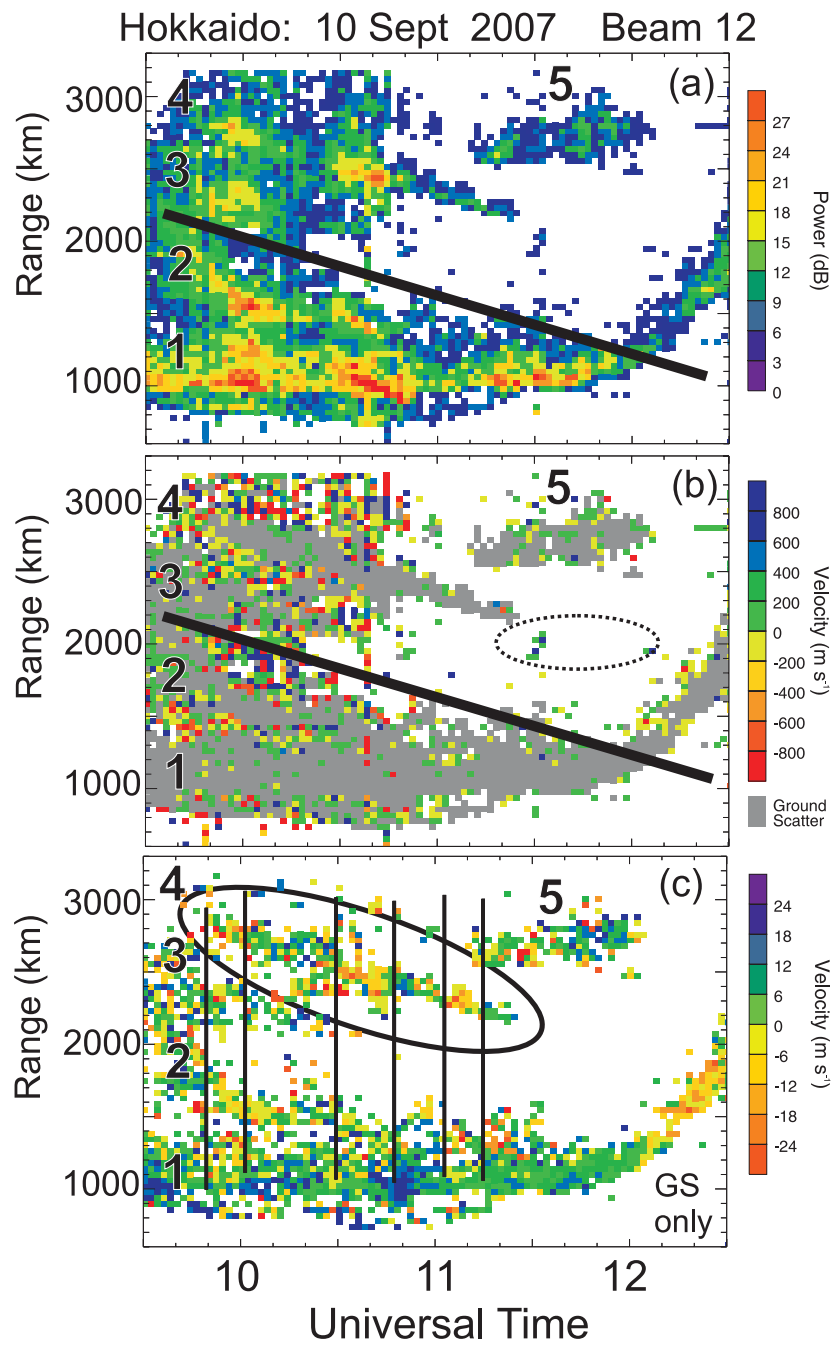

Fig. 3. (a) Power and $(\mathbf{b}, \mathbf{c})$ velocity of Hokkaido echoes in beam 12 between 09:00 and 12:30 UT on 10 September 2007. Two different scales for the velocity are used in panels (b) and (c). The sloped solid black line in panel (b) shows approximate location of the optical MSTID.

actual velocity values of the GS echoes; these are fairly low, as expected.

The radar echoes are dominated by GS and exhibit a complex pattern. There is an overall movement of GS echo bands towards lower latitudes between 09:30 UT and $\sim 11: 00-11: 30$ UT and towards higher latitudes after that. The observed dynamics would imply consecutive increase and decrease of the $\mathrm{F}$ layer electron density. This assumption is supported by the data from the ionosonde at Wakkanai (http://wdc.nict.go.jp/ISDJ/index-E.html) located close to the Hokkaido radar and by the overall decrease in GS echo occurrence after $\sim 11: 30$ UT. The SNR intensifications at $\sim 10: 00,10: 45$ and 11:30 UT across all ranges present another artifact occurring due to the periodical changes in the radar sensitivity, which was fixed later in 2007. 


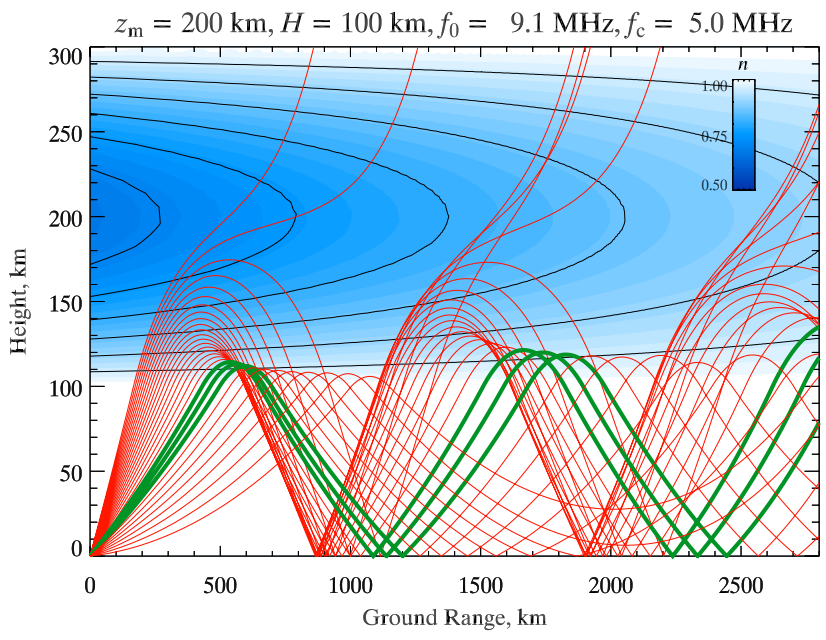

Fig. 4. Ray tracing of HF radio waves for the background conditions close to the ones for the 10 September 2007 event.

For the first half of the event, the GS signals can be divided into 4 different bands, which we labeled in Fig. 3 by numbers from 1 to 4 . Band 1 at $1000-1200 \mathrm{~km}$ was stable in range until $\sim 11: 00 \mathrm{UT}$ and then started to slowly move poleward. Band 2 at $\sim 1500 \mathrm{~km}$, poleward of band 1, was shifting towards the radar. It weakened after $\sim 11: 00 \mathrm{UT}$ and "merged" with band 1 . Band 3 at $\sim 2300 \mathrm{~km}$ disappeared after 10:45 UT. This band seemed to shift equatiorward as well. Finally, band 4 was clearly seen as a narrow stripe for $\sim 2 \mathrm{~h}$ continuously shifting equatorward $\sim 3000 \mathrm{~km}$ at 10:00 UT to $\sim 2300 \mathrm{~km}$ at 11:30 UT. This echo band is of prime interest in this study.

We also noted that between $\sim 11: 10$ and 12:10 UT, a patch of GS echoes was seen at ranges 2600-2800 km (band 5), which showed no apparent range progression. Data for other beams, e.g. beam 11, show existence of a patch of echoes within the time-range interval indicated by the dotted ellipse in Fig. 3b. The band 5 echoes are very likely 2-hop ground scatter formed by the unperturbed ionosphere (no MSTID involvement) at heights near the $\mathrm{F}$ region maximum (these heights were not involved in formation of the band 1-4 echoes).

For the equatorward-progressing band 4, Fig. 3c shows that the Doppler velocity was changing its polarity several times. There is a certain synchronism between band 4 and band 2 velocities illustrated by the vertical lines in Fig. 3c delineating the periods of echoes with the same velocity polarity

The above preliminary interpretation of HF propagation modes is based on numerical ray tracing performed for the unperturbed ionospheric conditions resembling those during the observations. Figure 4 illustrates typical paths of radio waves in the ionosphere for conditions typical of the observations. The ionosphere was modeled by a parabolic density profile characterized by a critical frequency of $f_{c r}=$
$5 \mathrm{MHz}$ at the radar location. It assumed to have $200-\mathrm{km}$ height span and the maximum at $200 \mathrm{~km}$. The ionosphere in Fig. 4 is shown in terms of the HF refraction index $n=$ $\left(1-\left(f_{c r} / f\right)^{2}\right)^{1 / 2}$, where $f$ is the radar frequency $(9.1 \mathrm{MHz}$ in our case). The neighboring contours (black) differ by $2.5 \%$, and the color scheme for the index of refraction is presented in the top right corner. To model the expected decrease in the electron density with increasing latitude, a negative gradient of the critical frequency of $15 \%$ per $1000 \mathrm{~km}$ was introduced (blue color becomes lighter at the far radar ranges). In Fig. 4, the first- and second-hop GS echoes, where the focusing occurs, are present at ground ranges of $\sim 1000 \mathrm{~km}$ and $\sim 2000 \mathrm{~km}$, respectively, and the respective group ranges would be some $\sim 200-300 \mathrm{~km}$ larger so that they would be consistent with ranges of band 1 and probably band $3 \mathrm{GS}$ echoes. Signatures of GS enhancement at ranges $\sim 1200 \mathrm{~km}$ and $2400 \mathrm{~km}$ corresponding to bands 2 and 4 are not seen. In Fig. 4 we marked by green color three rays that will be discussed in the next section; we will show that these rays experience significant focusing for a case of an electron density depletion being added to the regular ionosphere.

At this stage we can only confidently interpret bands 1 and 3 as the first- and second-hop GS modes, respectively. The negative velocity polarity for band 1 after $\sim 12: 10$ UT is consistent with our hypothesis that the ionosphere was experiencing general density decrease with upward motion of the electron density iso-contours after this time. Here we assume that positive (negative) polarity of the GS echo velocity can be interpreted as the downward (upward) motion of the ionosphere at the mid-way point of reflection.

Finally, the sloped thick black line in Fig. 3a, b represents an approximate location of the airglow depletion. While there are no intense radar echoes co-located with the airglow perturbation, there is a remarkable similarity in the rate of its equatorward progression and the band 4 dynamics. We suggest that that these are two independent signatures of MSTID propagation through the ionosphere, and we will analyze them in detail in the following section.

\section{Maps of the airglow perturbation and HF echo power}

To investigate the relationship between the optical and radar signatures of propagating perturbation in more details, we consider 2-D maps of the airglow relative intensity (deviation from mean values) and radar echo power, Fig. 5. The airglow intensity distribution is mapped in geographic coordinates, and positive (negative) deviation of the airglow is denoted by white (black) color. One can recognize a dark patch of decreased airglow in the middle of the images progressing equatorward; this patch represents a 2-D counterpart of the dark blue stripe in Fig. $2 b$.

The Hokkaido radar FoV is outlined by red color in Fig. 5. Since paths for the GS echoes include double reflection from the ionosphere, the radar echo ranges in Fig. 5 are made 3/4 


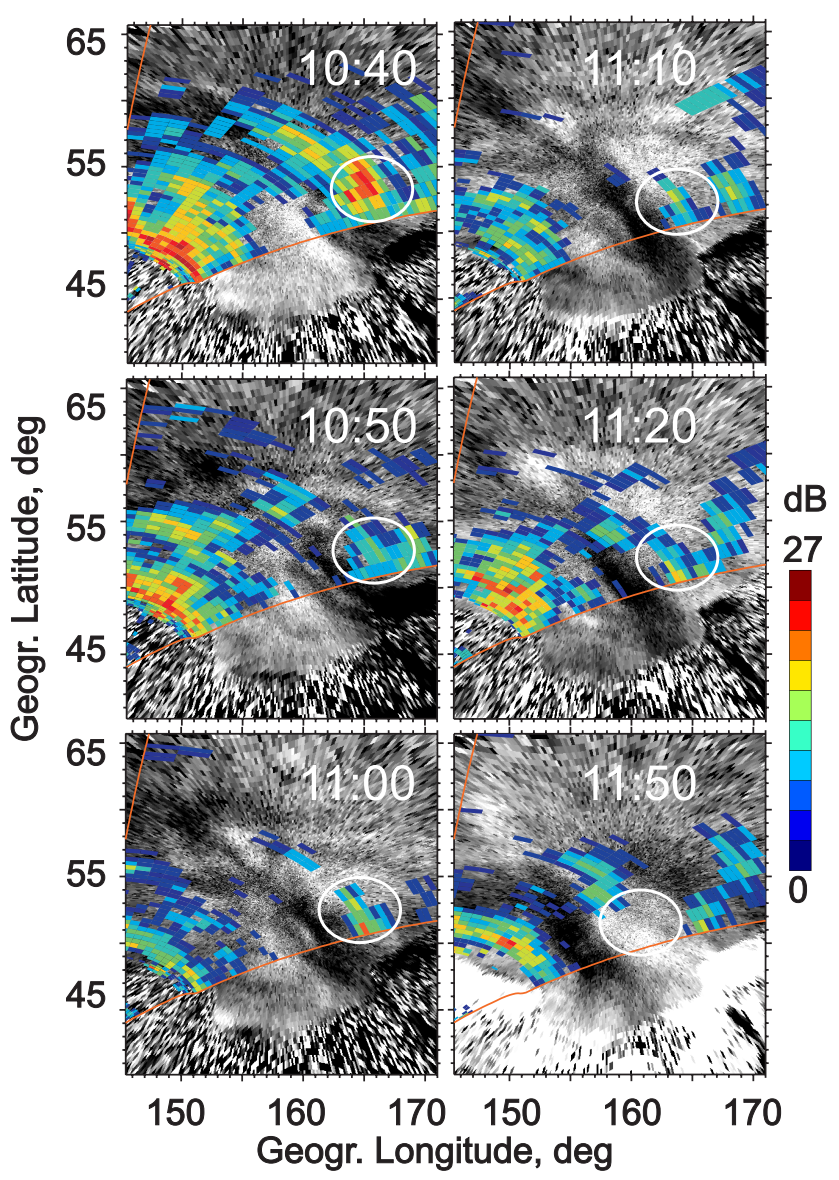

Fig. 5. A series of maps (between geographic latitudes of $40^{\circ}$ and $65^{\circ}$ and geographic longitudes of $145^{\circ}$ and $170^{\circ}$ ) for the airglow deviation from the 1-h running averages (in \%, dark is airglow depletion) and corresponding echoes observed by the Hokkaido radar. The echo ranges are scaled down by a factor of $3 / 4$. The luminosity and echo height were assumed to be $300 \mathrm{~km}$.

of the actual ranges for the echoes. This is done for convenience of presentation, and will be discussed more in the next section. To facilitate the comparison, the GS echoes that are assumed to be a radar analog of the optical depletion are encircled in white. There are no depletion-related radar echoes in the last frame, and the circle shows their expected location. Here, the echoes poleward of the circle correspond to the band 5 echoes seen in Fig. 3.

In Fig. 5 the dark luminosity patch is clearly progressing towards the radar. The respective HF echo progression is not so pronounced because the echo ranges were scaled down. The frame sequence covers the final period of band 4 echo existence. All plots show that the echoes are located somewhat poleward of the dark luminosity blob, but close to it.

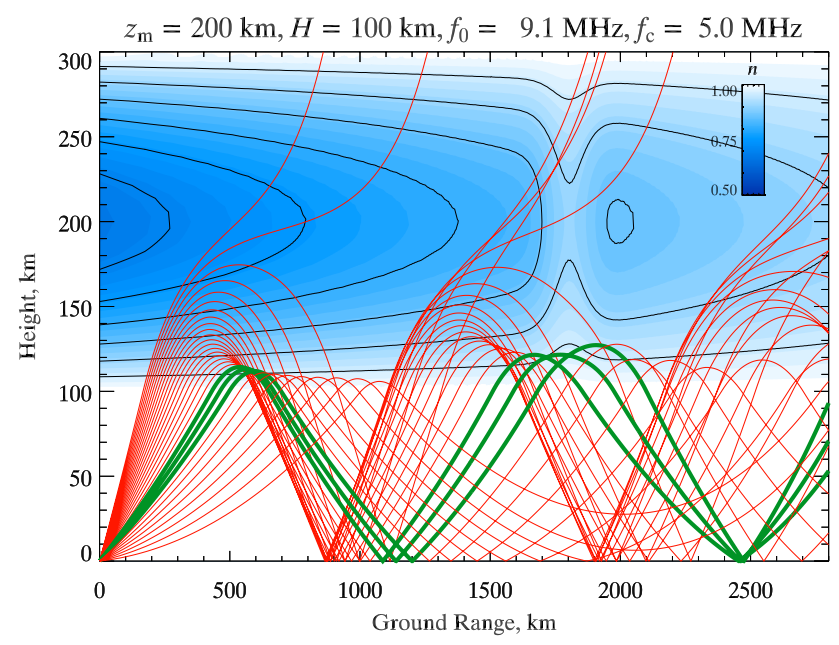

Fig. 6. Ray trajectories for the background ionosphere with added localized perturbation of the electron density. The resultant distribution of the refractive index for HF waves is shown by color and by contours that are $2.5 \%$ apart.

\section{Modeling of $\mathrm{HF}$ echo occurrence pattern for propa- gating MSTID}

A clear similarity between the rate of equatorward progression of airglow perturbation and band 4 GS radar echoes argues for their common source. We envision a scenario considered by Shiokawa et al. (2003b) and assume that the MSTID is produced by a localized perturbation $(\sim 200 \mathrm{~km})$ in the electric field propagating through the ionosphere from the north-east to the south-west. The direction of the electric field in the perturbation is assumed to be towards north-east; electric field of this orientation generates an upward motion of the ionospheric plasma. The upward motion of the plasma leads to a decrease in the airglow intensity, as discussed by Shiokawa et al. (2003b). In addition to that, a local plasma depletion is formed so that iso-contours of the electron density are distorted which would affect the radio ray trajectories in the ionosphere.

In our simulation, the electron density perturbation associated with MSTID is modeled by a Gaussoid of $\sim 200 \mathrm{~km}$ width, according to the observational widths of the airglow stripe in Fig. 2. The amplitude of perturbation was set to $20 \%$ in plasma frequency, consistent with expectations of Shiokawa et al. (2003b). The effect of this perturbation addition to the background distribution of the electron density (Fig. 4) is seen in Fig. 6 at ranges $1700-1800 \mathrm{~km}$ as a region where the density/refraction index iso-contours are "pinched" and the color is lighter.

In Fig. 6, ray paths are shown for the rays with the same elevation angles as in Fig. 4. The presence of density (refractive index) perturbation in the signal-forming area produces an additional ray focusing at ground ranges of $\sim 2500 \mathrm{~km}$, which is identical to the focusing effect caused 


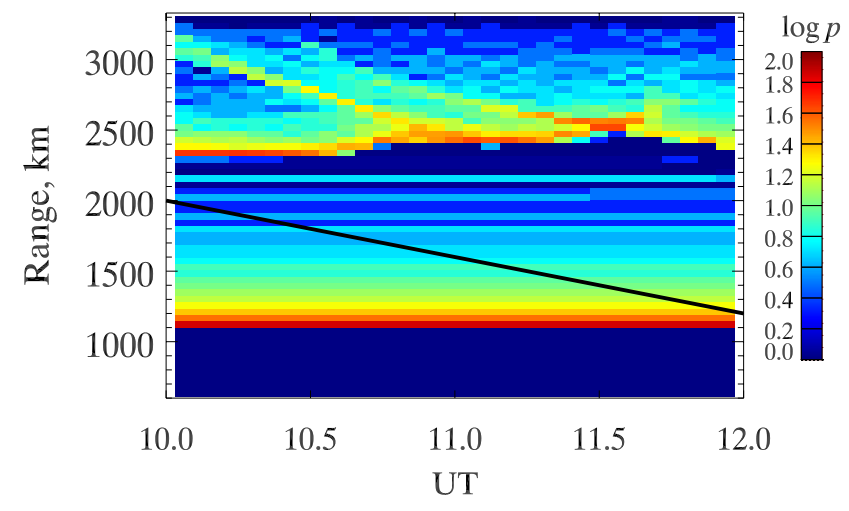

Fig. 7. Model distribution of HF echo power versus range for the ionospheric conditions of Fig. 5 and radar frequency of $9.1 \mathrm{MHz}$. The tilted black line roughly corresponds to the location of the center of the refractive index perturbation.

by "conventional" TIDs except there is no defocusing caused by the increases in the electron density. We highlight these rays by green color. The same rays in the "unperturbed" ionosphere (Fig. 4) show no focusing effect. Thus, for the selected position of the electron density depletion, an additional band of GS signals is expected at ranges $\sim 2500+200 \mathrm{~km}$. The expected GS forming area co-locates with the depletion region reasonably well, Fig. 5.

To make a detailed quantitative assessment of the GS echo intensity distribution with range for each instant of time, we performed ray tracings with step in elevation angle of $0.1^{\circ}$ for each location of the density perturbation and computed a number of trajectories that would fall into a specific bin of the group range (radar range gate) at the ground level. The size of the bin was selected to be $45 \mathrm{~km}$, according to the spatial resolution of the radar measurements.

Results of the ray tracing analysis for the 10:00-12:00 UT interval are presented in Fig. 7. Here the vertical and horizontal axes correspond to those in Fig. 3 and the location of the center of the density perturbation is shown by the solid black line. The color map corresponds to the logarithm of the number of rays at each range bin.

In Fig. 7 one can recognize strong signals at ranges of $\sim 1200 \mathrm{~km}$ and $\sim 2400 \mathrm{~km}$ which are the GS signals received through one- and two-hop propagation in the regular, unperturbed ionosphere (bands 1 and 3 in Fig. 3). A distinct feature of Fig. 7 is the tilted stripe of signals between $3000-2500 \mathrm{~km}$ progressing equatorward (towards the radar). These echoes originated from the additional focusing associated with the MSTID-produced perturbation of the electron density (green rays in Fig. 6). They closely resemble the observed dynamics of the band 4 GS echoes shown in Fig. 3. Importantly, the expected temporal displacement of these echoes is very similar to the equatorward progression of the optical feature (black sloped line). As time progresses, these echoes reach ranges of the second-hop skip zone for the background density dis- tribution. At this stage, the second band of the equatorward progressing echoes becomes apparent just poleward of the first one. The detailed analysis of individual trajectories revealed that the closer band is produced through focusing of the lower-elevation angle rays, while the weaker one corresponds to focusing of the higher elevation-angle (Pedersen) rays. Finally, both bands come together at $\sim 11: 30$ UT and form a modified skip zone at ranges farther than the one for the unperturbed situation. One would expect this effect because the electron density was depleted within the perturbation. We hypothesize that band 5 in Fig. 3 may correspond to the above-mentioned modified second-hop skip zone echoes. The experimental data in beam 12 do not show the Pedersenray related component progression probably because of its relative weakness, but such echoes can be seen in other radar beams (data are not shown).

In Fig. 3, starting from $\sim 12: 00$ UT, one cannot identify the separate band(s) of echoes except the first-hop band 1 quickly progresses away from the radar indicating that the electron density was probably gradually decreasing across the entire ionosphere. We relate the band 3 echo disappearance with the reduction of the electron density. A proper selection of time variation in the electron density maximum would allow one to simulate this effect too, but it lies outside the scope of this paper.

Finally, the modeling performed did not reproduce band 2 GS echoes (Fig. 3). These echoes occurred at somewhat larger ranges than the 1-hop band 1 echoes. At the beginning of the event, the band 2 echoes were gradually shifting equatorward and eventually "merging" with the band 1 echoes. We assume that perhaps this reflects some features in the electron density distribution with height which were not "captured" by the model (i.e., localized presence of distinct $F 1$ and $F 2$ layers).

\section{Discussion and summary}

In this study we presented Hokkaido HF radar observations of a perturbation in the ionosphere that was associated with the airglow perturbation propagating through the radar FoV. The optical feature was seen as a patch of depleted airglow intensity elongated in the north-west to south-east direction. The perturbation was progressing from north-east to southwest, which is typical of nighttime MSTID propagation for observations near Japan (e.g., Saito et al., 1998; Kubota et al., 2000). The event lasted for $\sim 3 \mathrm{~h}$.

The radar signature of the optical perturbation was a band of GS echoes observed at the Hokkaido radar far ranges (high latitudes) that was also moving equatorward synchronously with its optical counterpart. This progression lasted for about two hours, which is very unusual for these very different phenomena. We hypothesized that the optical and radar features are manifestations of a common source propagating through the ionosphere. Such source was proposed to be a 
medium-scale localized electric field structure. We assumed that the electric field would displace the $\mathrm{F}$ region plasma upward and produce negative perturbation in the airglow colocated with the area of "lifted up" electron density isocontours. This, in turn, would affect radio wave propagation through the perturbed ionosphere.

To test this hypothesis, we performed ray tracing modeling of the expected ranges of GS echoes for ionospheric conditions observed during the event. We found that the observed HF signature of MSTID propagation can be reproduced by introducing a medium-amplitude $(20 \%)$ localized $(\sim 200 \mathrm{~km})$ perturbation of the plasma frequency.

An interesting aspect of these observations, that has not been discussed so far, is the Doppler velocity of the GS echoes. According to the model, the rays that were focused near the depleted ionosphere traversed the leading and trailing parts of the density perturbation (Fig. 6). The ionospheric plasma is effectively moving up (down) at the leading (trailing) edge of the density depletion. The resultant velocity of the echoes would then depend on the relative contribution of signals from each edge and might be expected to compensate each other, at least partially. The observed velocity should be affected by the regular equatorward motion (positive Doppler shift) of the depleted region. However, the observations in Fig. $3 \mathrm{c}$ show that the Doppler velocity of the band 4 echoes was varying only between $\pm 20 \mathrm{~m} / \mathrm{s}$ whereas the equatorward motion is about $180 \mathrm{~m} / \mathrm{s}$. However, if the ionospheric reflection takes place near where the electron density contours are relatively horizontal (as they are for the green rays in Fig. 6) this equatorward motion will not affect the path lengths and hence will not be seen in the Doppler shift. There remains the question of what causes the small $\pm 20 \mathrm{~m} / \mathrm{s}$ which was positive roughly half of the time and during the other half time it was negative. In understanding this result, another factor might play an important role. Previously we indicated that there is a great deal of similarity between variations of Doppler velocity in bands 2 and 4 (Fig. 3c) which hints on the fact that, for the band 4 echoes received through 2-hop propagation path, the Doppler velocity was modulated by the large-scale plasma motion near the region of reflection for the first hop.

The source of the MSTID/electric field perturbations for the event under study is difficult to identify. On 10 September 2007 the $K_{p}$ index was 1- and 0+ between 09:00 UT and 15:00 UT, implying very quiet conditions. Very little magnetic activity was seen in the Alaskan sector of the Arctic where a potential source can be located, based on the direction of the optical feature propagation. Therefore, the scenario proposed by Huang et al. (1998) is not applicable in this case. Influence of solar wind sources such as those driving the gravity waves (Prikryl et al., 1995) is highly improbable for an isolated disturbance. Azimuthal progression of airglow perturbation by at least $25^{\circ}$ in longitude (effective size of the Paratunka FoV) in $\sim 3 \mathrm{~h}$ would favor involvement of the semidiurnal tide. Neutral wind motions do influence the airglow intensity (Shiokawa et al., 2003b), but quantitative assessment of this scenario is impossible due to the lack of necessary data. Thus, we will continue searching for other events that might provide extra information on the sources of MSTID in airglow and HF radar echoes.

In summary, the results of this study are the following:

1. A localized depletion of airglow intensity propagating from the North-East to the South-West has been identified in OMTI camera data collected at Paratunka (Kamchatka, Russia) on 10 September 2007 between $\sim$ 10:00 and 14:00 UT. The perturbation extended from the north-west to the south-east direction. The concurrently operated Hokkaido HF radar detected a highly localized GS echo band that can be associated with the passage of this optical perturbation. The band was moving equatorward synchronously with the equatorward progression of the airglow perturbation. The Doppler velocity of the echoes was varying between $\pm 20 \mathrm{~m} / \mathrm{s}$.

2. Both the progressing airglow perturbation and its radar signature are suggested to result from passage of a localized electric field structure through the common viewing area. The electric field vector in the structure was assumed to be towards north-east, i.e. opposite to the structure propagation direction. For such orientation of the electric field, the ionospheric plasma would be lifted up leading to a negative perturbation in the airglow intensity and electron density depletion so that a separate GS radar echo component is formed through additional HF ray focusing. The observed Doppler velocity of the GS echoes was found to be variable, which seems to be caused by vertical plasma motions in the ionospheric region in the vicinity of the first hop reflection point.

Acknowledgements. This study was supported by Grants-in-Aid for Scientific Research (16403007; 18403011; 19340141; 19403010; 20244080) from the Ministry of Education, Culture, Sports, Science and Technology of Japan, and by Project 2 of the Geospace Research Center, Solar Terrestrial Environment Laboratory. AVK acknowledges the Solar-Terrestrial Environment Laboratory of Nagoya University for funding during his stay in Japan. This work was also supported by NSERC (Canada) grant to AVK.

Topical Editor M. Pinnock thanks E. Afraimovich and another anonymous referee for their help in evaluating this paper.

\section{References}

Chisham, G., Lester, M., Milan, S. E., Freeman, M. P., Bristow, W. A., Grocott, A., McWilliams, K. A., Ruohoniemi, J. M., Yeoman, T. K., Dyson, P. L., Greenwald, R. A., Kikuchi, T., Pinnock, M., Rash, J. P. S., Sato, N., Sofko, G. J., Villain, J.P., and Walker, A. D. M.: A decade of the Super Dual Auroral Radar Network (SuperDARN): scientific achievements, new techniques and future directions, Surv. Geophys., 28, 33-109, doi:10.1007/s10712-007-9017-8, 2007. 
Bristow, W. A., Greenwald, R. A., and Samson, J. C.: Identification of high-latitude acoustic gravity waves sources using the Goose Bay HF radar, J. Geophys. Res., 99, 319-331, 1994.

Garcia, F. J., Kelley, M. C., Makela, J. J., and Huang, C.-S.: Airglow observations of mesoscale low-velocity traveling ionospheric disturbances at midlatitudes, J. Geophys. Res., 105, 18407-18415, 2000.

Greenwald, R. A., Baker, K. B., Dudeney, J. R., Pinnock, M., Jones, T. B., Thomas, E. C., Villain, J.-P., Cerisier, J.-C., Senior, C., Hanuise, C., Hunsuker, R. D., Sofko, G., Koehler, J., Nielsen, E., Pellinen, R., Walker, A. D. M., Sato, N., and Yamagishi, H.: DARN/SuperDARN: A global view of the dynamics of highlatitude convection, Space Sci. Rev., 71, 763-796, 1995.

Huang, C-S, Andre, D., and Sofko, G. J.: Observations of solar wind directly driven auroral electrojets and gravity waves, J. Geophys. Res., 103, 23347-23356, 1998.

Kelley, M. C. and Makela, J. J.: Resolution of the discrepancy between experiment and theory of midlatitude F-region structures, Geophys. Res. Lett., 28(13), 2589-2592, 2001.

Kubota, M., Shiokawa, K., Ejiri, M. K., Otsuka, Y., Ogawa, T., Sakanoi, T., Fukunishi, H., Yamamoto, M., Fukao, S., and Saito, A.: Traveling ionospheric disturbances observed in the OI 630$\mathrm{nm}$ nightglow images over Japan by using a multi-point imager network during the FRONT campaign, Geophys. Res. Lett., 24, 4037-4040, 2000.

MacDougall, J. W., Andre, D. A., Sofko, G. J., Huang, C.-S., and Koustov, A. V.: Travelling ionospheric disturbance properties deduced from Super Dual Auroral Radar measurements, Ann. Geophys., 18, 1550-1559, 2000, http://www.ann-geophys.net/18/1550/2000/.

Nishitani, N., Ogawa, T., Kikuchi, T., Kataoka, R., Hosokawa, K., Yamagishi, H., and Yukimatu, A. S.: Initial observations with the Hokkaido radar, Paper presented at the Annual SuperDARN Workshop, 4-8 June, Abashiri, Hokkaido, Japan, 2007.
Nishitani, N., Ogawa, T., and Kikuchi, T.: Hokkaido HF radar: progress report on mid-latitude SuperDARN radar in East Asia, American Geophysical Union, Fall Meeting 2005, abstract \#SA23A-0300, 2005.

Perkins, F.: Spread F and ionospheric currents, J. Geophys. Res., 78, 218-226, 1973.

Ponomarenko, P. V., Waters, C. L., and Menk, F. W.: Effects of mixed scatter on SuperDARN convection maps, Ann. Geophys., 26, 1517-1523, 2008, http://www.ann-geophys.net/26/1517/2008/.

Prikryl, P., Muldrew, D. B., Sofko, G. J., and Ruohoniemi, J. M.: Solar wind Alfvén waves: a source of pulsed ionospheric convection and atmospheric gravity waves, Ann. Geophys., 23, 401417, 2005, http://www.ann-geophys.net/23/401/2005/.

Saito, A., Fukao, S., and Miyazaki, S.: High resolution mapping of TEC perturbations with the GSI GPS network over Japan, Geophys. Res. Lett., 25, 3079-3082, 1998.

Samson, J. C., Greenwald, R. A., Ruohoniemi, J. M., and Baker, K. B.: High-frequency radar observations of atmospheric gravity waves in the high-latitude ionosphere, Geophys. Res. Lett., 16, 875-878, 1989.

Shiokawa, K., Ihara, C., Otsuka, Y., and Ogawa, T.: Statistical study of nighttime medium-scale traveling ionospheric disturbances using midlatitude airglow images, J. Geophys. Res., 108(A1), 1052, doi:10.1029/2002JA009491, 2003a.

Shiokawa, K., Otsuka, Y., Ihara, C., and Ogawa, T.: Ground and satellite observations of nighttime medium-scale traveling ionospheric disturbance at midlatitude, J. Geophys. Res., 108, 1145, doi:10.1029/2002JA009639, 2003b.

Shiokawa, K., Otsuka, Y., and Ogawa, T.: Propagation characteristics of nighttime mesospheric and thermospheric waves observed by optical mesosphere thermosphere imagers at middle and low latitudes, Earth, Planets and Space, 61, 479-491, 2009. 